\section{Runs of correct and incorrect predictions}

\section{as determinants of choice reaction time*}

\author{
CHARLES P. WHITMAN and E. SCOTT GELLER $†$ \\ Virginia Polytechnic Institute and State University, Blacksburg, Va. 24061
}

Intrasequential effects of prediction outcomes on choice reaction speed (CRS) were studied in a two-alternative reaction task. Prior to each of 300 stimulus presentations, Ss predicted the next stimulus; following each presentation, Ss identified the stimulus by pressing one of two reaction triggers. Not only was CRS faster to predicted than nonpredicted events, but given a correctly or incorrectly predicted event, CRS was significantly faster when the preceding prediction outcomes were correct than when they were incorrect. The implications of these results for dichotomous and continuous notions of expectancy are discussed.

Choice reaction time (CRT) studies in which Ss make a stimulus prediction before each stimulus is presented for identification have demonstrated that Ss respond significantly faster to correctly predicted stimuli (e.g., Keele, 1969; Hinricks \& Krainz, 1970). Some CRT models which account for this effect of prediction outcome on CRT employ expectancy notions. Specifically, two alternative views of Ss' expectancy processes have been considered: a dichotomous notion (e.g., Falmagne, 1965) and a continuous notion (e.g., Geller \& Pitz, 1970). A dichotomous expectancy hypothesis assumes that $S$ is either "set" or "unset" for a given stimulus. However, the notion of a continuously variable expectancy process implies that S's readiness for a given stimulus varies in degree within a given "set" or "unset" state. Although a dichotomous expectancy theory provides a parsimonious explanation for the finding that Ss are faster to the expected or anticipated stimulus, the observation that CRT is significantly influenced by stimulus run length and probability, when prediction outcome is controlled, supports the notion that expectancy is a continuous process (Geller \& Pitz, 1970). The present study was designed to determine the intrasequential effects on CRT of runs of correct and incorrect prediction outcomes. The necessity of considering higher order sequential effects of Ss' prediction outcomes in CRT modeling would be indicated if prediction outcomes on trials preceding a given stimulus is found to influence CRT to that stimulus.

*This research was facilitated by college gift equipment contributed by Western Electric Company and the Chesapeake and Potomac Telephone Company. Special thanks are due to Richard F. Wrenn for constructing the logic equipment.

+ Requests for reprints should be sent to E. Scott Geller, Department of Psy chology. Virginia Polytechnic Institute and State University, Blacksburg, Va. 24061.
One theory of expectancy as a continuous process assumes that S's degree of expectancy for a given stimulus is a direct function of S's confidence (i.e., strength of belief) in his prediction (Geller \& Pitz, 1970). Furthermore, an increase in the degree of expectancy for a predicted stimulus a ugments both the response facilitation for the predicted stimulus and the response inhibition for the nonpredicted alternative. Assuming that S's confidence in a prediction is a direct function of the number of consecutive correct predictions, S's confidence and therefore his expectancy for the next stimulus should be increased following each correct prediction. Correspondingly, each consecutive incorrect prediction outcome should reduce S's confidence in his next prediction, and therefore lessen his expectancy for the predicted stimulus. Such a continuous expectancy hypothesis would predict the following relationships between CRT and the number of consecutive correct or incorrect stimulus predictions (i.e., prediction outcome run length). CRT to correctly predicted events preceded by runs of correct predictions should be an inverse function of run length, while CRT to correctly predicted events which are preceded by incorrect prediction runs should be a direct function of run length. On the other hand, given that the inhibition to respond to a nonpredicted stimulus increases following a correct prediction outcome and decreases following incorrect outcomes, CRT to incorrectly predicted stimuli should vary directly with the run length of preceding correct predictions and inversely with the run length of preceding incorrect predictions.

\section{SUBJECTS}

Twenty-two students (16 males, 6 females), none of whom had previously participated in a reaction time experiment, participated individually in the experimental sessions. All Ss were enrolled in introductory psychology classes at Virginia Polytechnic Institute and State University, and received optional research credit for their participation. The data of two male Ss were disregarded because each $\mathrm{S}$ made more than $5 \%$ errors (i.e., anticipations or incorrect identifications).

APPARATUS AND PROCEDURE

The symbols $U$ and $\cap$ (verbally labelled up and down, respectively) were presented by a $1 / 2 \times 2$ in. digital readout which was mounted behind a one-way mirror. During the session, the room was darkened such that Ss could not see through the mirror except when the readout was illuminated.

The two stimulus alternatives were presented an equal number of times during the 300 trials. Throughout the first 150 trials, $\cap$ occurred $70 \%$ of the time and $U$ occurred $30 \%$; on the remaining 150 trials, the relative frequencies were reversed (i.e., $\cap=30 \%, U=70 \%$ ). For each 150 trials, runs of the more probable stimulus never exceeded seven and runs of the least probable stimulus never exceeded three.

In the task instructions, $E$ emphasized speed and accuracy and indicated that the stimulus sequence had been preprogrammed on punched paper tape. The $S$ was not given any information concerning the relative frequencies of the stimulus alternatives. The initial 10 trials were considered practice and during these presentations S's questions concerning his task were answered. For each of the 310 trials, the order of events was: S's verbal prediction, a warning-buzzer, a random time interval of between .5 and $2 \mathrm{sec}$, a stimulus presentation, and S's choice reaction which turned off the stimulus. The latency between stimulus presentation and identification was measured to the nearest millisecond by a Hunter Model 120A Klockounter. S's identification response consisted of pulling one of two microswitches mounted in separate left- or righthand triggers. Ten Ss pulled the right trigger to identify $U$ and the left trigger following $\cap$. The reverse mapping relationship was used for the remaining $10 \mathrm{Ss}$.

\section{RESULTS}

To normalize the data and maximize differences at high performance levels, each latency was converted to a choice reaction speed $(\mathrm{CRS}=1 / \mathrm{CRT})$. The mean CRS for each of 12 prediction run categories was calculated for each S. Four major categories were determined by correct or incorrect current prediction outcomes immediately preceded by 


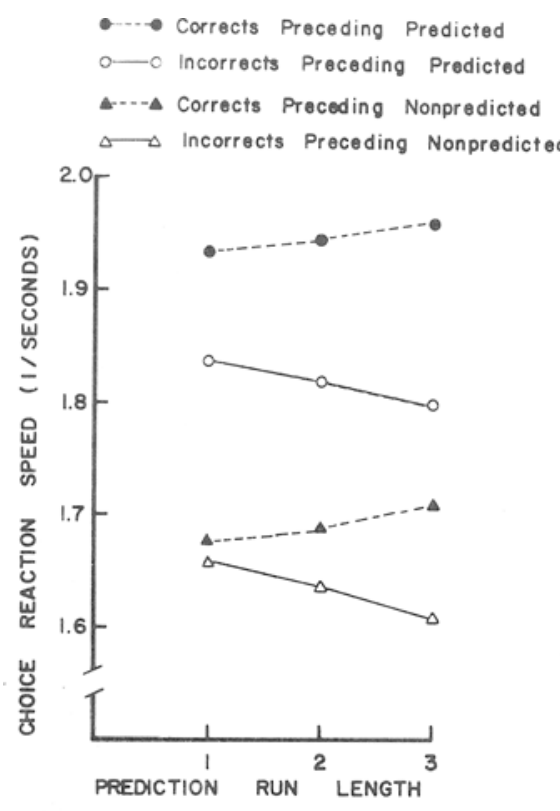

Fig. 1. Choice reaction speed as a function of the prediction run length preceding predicted and nonpredicted stimuli. The prediction run length refers to the number of consecutive correct or incorrect prediction outcomes preceding the identified stimulus.

correct or incorrect prediction outcomes; three subcategories within each major category were determined by the number of consecutive correct or incorrect predictions (i.e., prediction run length). Let the letters "P," for a "predicted stimulus," and "N," for a "nonpredicted stimulus." designate the outcome of the current prediction trial, and the letters " $\mathrm{C}$," for "correct prediction," and "I," for "incorrect prediction," designate the outcome of the immediately preceding trials. Then the four major categories with three subcategories each are: (1) predicted events preceded by correct prediction outcomes, including the subcategories CP, CCP, CCCP, (2) predicted events preceded by incorrect outcomes, including IP, IIP, IIIP, (3) nonpredicted events preceded by correct outcomes, including $\mathrm{CN}$, $\mathrm{CCN}, \mathrm{CCCN}$, and (4) nonpredicted events preceded by incorrect outcomes, including IN, IIN, IIIN. For example, the entry for each $S$ in Category IIP was the mean CRS following a predicted stimulus which had been preceded by two consecutive incorrect prediction outcomes. For each $\mathrm{S}$, there were at least five speeds in each category.

As Fig. 1 indicates, CRS was faster to predicted events than to nonpredicted events and, within both the predicted and nonpredicted event categories, CRS was faster when the prediction outcomes on preceding trials were correct than when they were incorrect.

The overall analysis of variance, a 2 (current prediction outcome) by 2 (preceding prediction outcome) by 3 (run length) factorial, indicated a main effect of current prediction outcome, $\mathrm{F}(1,19)=75.53, \mathrm{p}<.001$, and an interaction between current prediction outcome and preceding prediction outcome $F(1,19)=21.38, \quad p<.001$. The main effect of preceding prediction outcome did not reach significance at the .05 level, i.e., $F(1,19)=4.14, .10>p>.05$. Neither the main effect of run length nor the interactions with run length were significant, each $F<1$. The separate 2 by 3 analysis of variance for predicted events indicated a significant main effect due to the prediction outcome on preceding trials, $F(1,19)=25.92, \quad p<.001$, no significant effect of run length, $F<1$, but a significant interaction between the type of prediction outcome on preceding trials (correct vs incorrect) and the prediction run length, $F(2,38)=4.57, \quad p<.025$. Likewise, the 2 by 3 analysis of variance for nonpredicted events indicated a significant effect of preceding prediction outcome, $F(1,19)=4.98$, $\mathrm{p}<.025$, no significant effect of run length, $F<1$, and a significant Run Length by Preceding Prediction Outcome interaction, $F(2,38)=5.91$, $\mathrm{p}<.01$. As Fig. 1 indicates, the

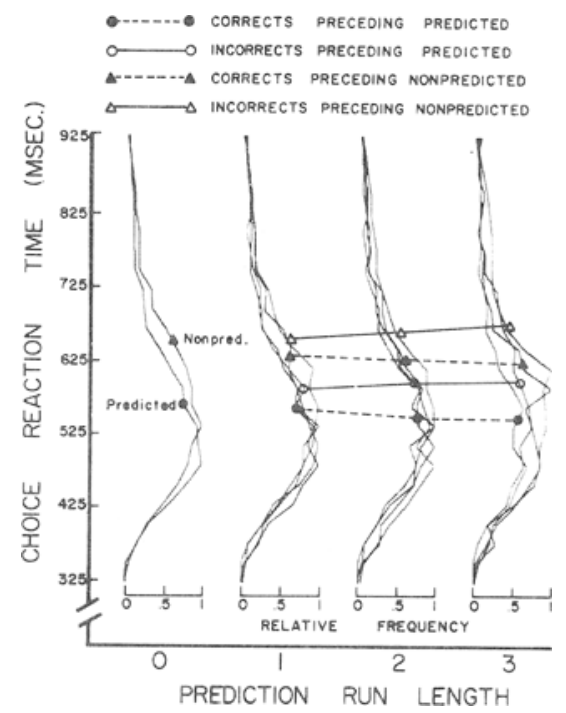

Fig. 2. Choice reaction time distributions and means as a function of the prediction run length preceding predicted and nonpredicted stimuli. The distributions in the first category (run length $=0$ ) represent the latencies following predicted or nonpredicted events, regardless of the preceding outcomes. interactions between prediction outcome and run length were due to a direct relationship between CRS and the number of consecutive correct predictions, and an inverse relationship between CRS and the number of consecutive incorrect predictions.

In order to demonstrate that the observed effects of prediction outcome on CRS occurred independently of stimulus probability, the prediction outcome sequences ending with the less probable stimulus alternative were omitted in a second categorization analysis. The observed relationships between CRS and runs of correct and incorrect prediction outcomes preceding the more probable stimulus were similar to the relationships in Fig. 1, which included those prediction runs which preceded the less probable stimulus. A comparison between the relationships obtained from each categorization method by a 2 (categorization method) by 2 (current prediction outcome) by 2 (preceding prediction outcome) by 3 (run length) analysis of variance indicated no significant interactions with categorization method (all Fs $<1$ ).

Since the "down" stimulus was presented more often during the initial 150 trials (i.e., $70 \%$ ) and then occurred on $30 \%$ of the latter 150 trials, Ss predicted "down" more often than "up" on the middle 100 trials of the session. Consequently, during the 50 trials prior to the probability reversal, runs of correct predictions frequently occurred; during the initial 50 trials following reversal, runs of incorrect predictions were prominent. However, although the reversal design had the advantage of influencing the occurrence of consecutive correct and incorrect prediction outcomes, the frequent occurrence of stimulus runs in a $70 \%-30 \%$ distribution of events introduces the possibility that the observed effects of prediction outcome could be confounded with response repetition effects. For example, if Ss consistently made long runs of predictions of the more probable stimulus, then runs of correct predictions would imply runs of the same stimulus, and, therefore, the direct relationship observed between CRS and the number of consecutive correct prediction outcomes preceding predicted and nonpredicted events could be explained by a response repetition effect (e.g., Bertelson, 1963). However, an additional analysis was done which indicated that the relative frequency of the least probable stimulus in each of Categories CCCP, IIIP, CCCN, and IIIN was $7 \%, 9 \%, 8 \%$, and $7 \%$, respectively. Thus, the number of response repetitions was approximately the same for each 
category, and therefore, the observed relationships occurred under equivalent response repetition conditions.

It is of theoretical importance to establish that the apparently continuous results presented in Fig. 1 are not a misrepresentation of a discrete function. That is, the continuous variation in the mean reaction speed of the various categories may be due to differences in the proportion of trials on which the $S$ is "set" in a discrete way. For this reason, an analysis of the distribution of reaction times in each of the categories was calculated and plotted by an IBM 360 computer system. As is evident in Fig. 2, the distribution of times in each category is relatively normal and is definitely unimodal. Although the distributions presented in Fig. 2 are summed over all Ss, the distributions for each $S$ were examined and also found to be unimodal.

\section{DISCUSSION}

The results presented in Fig. 2 are critical for the interpretation of Fig. 1 . That is, the singularity in mode of each distribution implies that there are, indeed, several discrete levels of expectancy or that expectancy is, in fact, a continuous dimènsion. Perhaps the notion of expectancy as a continuous dimension can provide the most parsimonious explanation of the present data.

In addition to replicating the earlier finding that Ss respond faster to predicted than to nonpredicted stimuli (e.g., Keele, 1969; Hinricks \& Krainz, 1970), the present study demonstrated intrasequential effects on CRS of the prediction outcomes which preceded an event. Specifically, third-order sequential effects were indicated by the significant interactions between the outcome of preceding predictions (correct vs incorrect) and the run length of that prediction outcome. The interaction between prediction outcome and run length preceding predicted stimuli confirmed the hypothesis generated by the continuous expectancy notion entertained herein. That is, according to the notion that expectancy is a continuous process, a run of correct prediction outcomes increased Ss' degree of expectancy for the next predicted stimulus, which in turn augmented the response facilitation to identify a correctly predicted stimulus. On the other hand, a run of incorrect prediction outcomes reduced Ss' degree of expectancy for the stimulus predicted and thus decreased the facilitation to identify a correctly predicted stimulus.

However, the observed interaction between prediction outcome and run length preceding nonpredicted stimuli was not as hypothesized. Therefore, a response facilitation notion should be expanded to explain the present finding that a preceding prediction outcome influences CRS to an incorrectly predicted stimulus. In other words, preceding correct predictions facilitate $S s^{\prime}$ reactions to subsequent stimuli, even though the stimuli may be nonpredicted; likewise, preceding incorrect prediction outcomes reduce response facilitation to nonpredicted stimuli.

Consequently, whether or not stimulus expectancy or response facilitation are appropriate mediating mechanisms for the present CRT task, it was apparent that Ss were not simply faster following correct predictions and slower following incorrect predictions; rather, given a correct or incorrect prediction, Ss' reaction speeds were influenced by the prediction outcomes on immediately preceding trials. Thus, the present results indicated that CRT models which describe prediction outcome effects must account for the fact that there are at least third-order sequential effects of prediction outcome.

REFERENCES

BERTELSON, $P$. $S-R$ relationships and reaction times to new versus repeated signals in a serial task. Journal of Experimental Psychology, 1963, 65, 478-484.

FALMAGNE, J. C. Stochastic models for choice reaction time with application to experimental results. Journal of Mathematical Psychology, 1965, 2, 77-124.

GELLER, E. S., \& PITZ, G. F. Effects of prediction, probability, and run length on choice reaction speed. Journal of Experimental Psychology, 1970, 84. 361-367.

HINRICKS, J. V., \& KRAINZ, P. L Expectancy in choice reaction time: Anticipation of stimulus or response? Journal of Experimental Psychology. $1970,85,330-334$.

KEELE, S. W. Repetition effect: A memory-dependent process. Journal of Experimental Psychology, 1969, 80, 243-248.

\title{
Encoding as a function of the presentation-rehearsal interval in short-term memory*
}

\author{
LAIRD S. CERMAK† and ROGER LEVINE \\ Tufts University, Medford, Mass. 02155
}

The Ss participated in a short-term memory distractor task in which the presentation-rehearsal interval was systematically varied. Three presentationrehearsal intervals were employed: a 2 -sec (2-0) visual presentation group, a 5-sec (5-0) visual presentation group, and a 2 -sec visual plus 3 sec of additional rehearsal time $(2-3)$ group. Both recall and recognition test results showed the 2-3 group's performance to be the same as the 2.0 group. Increased rehearsal time, without the TBR material present, did not result in an increased level of encoding, while increased rehearsal concurrent with increased visual presentation (the 5-0 group) did allow an increased complexity of encoding.

Anisfeld \& Knapp (1968) proposed that when a word is stored for future retrieval it is stored as a "feature complex." This complex is a composite of the word's physical, acoustic, and semantic characteristics. During the presentation of the word, the $\mathbf{S}$ engages in an analysis and a storing of the presented word on the basis of these characteristics.

It has been proposed further that these "features" of encoding should be thought of as a hierarchy of encoding "levels," the more complex the level, the more time it takes to encode on that level (Cermak, 1970). Physical characteristics are more readily

*This work was supported in part by a Tufts University Faculty Grant, No. BD402U, to the senior author.

tRequests for reprints should be sent to Laird S. Cermak, Department of Psychology. Tufts University, Medford, Mass. $02155^{\circ}$ encoded than are the acoustic, which in turn take less time than the semantic. Consequently, the more time the $S$ has to encode a word, the higher the level of encoding he is likely to achieve and the more precision he can attain within that level of encoding.

Several studies have demonstrated that the longer the presentationrehearsal interval in an STM distractor task, the greater the retention (e.g., Peterson \& Peterson, 1959; Pollack, 1963; Miller, 1970). However, in all these cases, the $S$ was either instructed to repeat the TBR item a specified number of times or the material was present visually for the entire interval. Research of this type has always assumed that the $S$ actively processes the information throughout the entire interval and that the increased processing time results in increased recall probability. The purpose of the 